\title{
A Novel Multichannel Access Scheme with Directional Antennas in WLANs
}

\author{
Yuan Cheng, Suming Jiang, Lele Song \\ State Grid Electric Power Research Institute \\ Nanjing, China \\ e-mail: chengyuan6@sgepri.sgcc.com.cn
}

\begin{abstract}
Directional antennas in wireless local area networks (WLANs) offer more benefits than traditional omni-directional antennas. Use of directional antenna can increase the spatial reuse and largely reduce the radio interference. In this paper, we attempt to propose a new medium access control (MAC) protocol utilizing the directional antennas and the multichannels access mechanism without any acknowledgement of location information of the nodes in the network. Simulation results show that our scheme performs better than IEEE 802.11, which produces significant improvements from system throughput, average access delay and packet dropping rate over the omni-directional communications with single channel.
\end{abstract}

Keywords-directional antennas; multichannel; medium access control; WLANs

\section{INTRODUCTION}

In wireless communication networks, MAC protocols are used to manage the access of a shared channel. As the medium access schemes may significantly affect the overall performance of a wireless network, careful design of the MAC protocols is crucial to ensure efficient operation of a network.

Directional antennas have a number of advantages over omni-directional antennas in WLANs. By focusing energy only in the intended direction, directional antennas can increase spatial reuse and provide longer transmission ranges. Reduced interference and longer range translate into higher network capacity, which means more simultaneous transmissions are supported and fewer hops are needed. However, the use of directional antennas on mobile terminals introduces the complex issue of finding the desired direction for transmission or reception. This issue is particularly critical in the wireless local area network which has no centralized control. The mobile terminals may have limitation of size and the complexity of communication hardware. The design of the IEEE 802.11 implicitly assumes an omni-directional antenna at the physical layer. Several MAC protocols have been proposed that are suitable for 802.11 with beamforming antennas [1],[2],[3]. Ko et al. [4] proposed to transmit a RTS directionally, only if the RTS does not collide with other ongoing communications. Kobayashi and Nakagawa [5] proposed a similar mechanism in which NAVs are assigned.

In order to reduce packet collisions and to increase the system throughput, some researchers proposed to divide the single channel into several sub-channels. One is served as a control sub-channel and the rest are served as data subchannels. The control sub-channel is used to reserve the data sub-channel for the following data transmission. Such reservation can be performed through the RTS/CTS (ReadyTo-Send/Clear-To-Send) dialogue. Examples of the multichannel MAC protocols are [6]-[9]. The use of multichannel access, which decentralizes the contentions in a single channel to several separated sub-channels, can reduce the packet collisions so as to increase utilization of frequency spectrum.

Due to the superiorities of directional antennas and the mechanism of multichannel access, we are inspired to apply them to the current 802.11 MAC protocol. It should be noted that the novel proposed multichannel access scheme using directional antennas is based on the assumption that the location of each node is unknown in advance. That is the main difference between our new scheme and the previous work done by other researchers. Although the knowledge of the location of the nodes can be carried out by GPS, the cost of facilities is increased. The new scheme not only improves performance of the network, but also reduces the complexity of the equipment.

The rest of this paper is organized as follows. Section II describes the proposed multichannel access scheme using directional antennas in details. Section III presents the simulation results of the proposed scheme. Finally, conclusions are drawn in Section IV.

\section{Multichannel AcCESS Scheme Using DIRECTIONAL ANTENNAS}

\section{A. System Assumptions}

There are several assumptions in our study. Each node in the network is stationary and only has information about its own location. Transmission power is high enough so that each node can communist with other one in the wireless network using single-hop mode. Directional antennas are equipped for each node. The real-time status of each subchannel for all directions can be sensed. In this paper, we concentrate on the performance evaluation of our new scheme operated by distributed coordination function (DCF) mode [10].

\section{B. Directional Network Allocation Vector}

The 802.11 MAC protocols are based on virtual carrier monitoring. In order to avoid packet collisions, network allocation vectors (NAVs) are used for recording the utilization of the channels in previous research work. The value of NAVs is renewed according to the contents in RTS and CTS packets. NAVs indicate the time period that should be delayed for its packet transmission. Because directional 
antennas and multichannel access mechanism are applied in our scheme, NAVs could not wholly describe the conditions of channel occupation and spatial utilization. As a result, NAVs should be redefined in the proposed scheme.

For one thing, the characteristics of directions should be added to the NAVs so as to describe the spatial usage. For another, each node needs to maintain an individual NAV for each sub-channel and renew it timely. Therefore, a two dimension Directional Network Allocation Vectors (DNAVs) emerges as the times require. The DNAV table is an $N^{*} M$ matrix, where $N$ equals to the number of directional antennas and $M$ equals to the number of sub-channels. The element in the DNAV table represents the time period the node should wait for on a certain sub-channel and a certain direction before its data transmission. The setting and renewing procedures of the DNAV table are clarified in the next part.

\section{Detailed Description of The New Scheme}

Firstly, we discuss the process of data transmissions in the four-way handshaking mode between a pair of nodes with the proposed multichannel access scheme using directional antennas. We denote the source node as $\mathrm{S}$ and the destination node as $\mathrm{D}$.

\section{1) RTS Sending}

When node $S$ receives a data packet from the link layer, it sends an RTS frame to the physical layer, including the location information of its own and the identification of its destination node. At the same time, there is a DNAV table recording the carrier sensing results for each direction of arrival (DoA) on each sub-channel. If data transmission is detected on a certain sub-channel for a certain direction or a RTS frame sent by other node is received, this direction on this sub-channel is unavailable. If all the directions for a certain sub-channel are unavailable, it means the sub-channel is busy, and hence is unavailable.

Because node $\mathrm{S}$ does not know the location of node $\mathrm{D}$, it is unable for node $\mathrm{S}$ to select the proper directional antenna to send the RTS frame. In our scheme, node $S$ always selects the sub-channel that has the most available directions to transmit a RTS frame according to the DNAV table. It is evident that sending the RTS frame on the sub-channel which has the most available directions may increase the probability of successful reception for node D.

\section{2) RTS Receiving and CTS Sending}

When Node D is idle, it senses each sub-channel all the time with omni-directional mode. If it receives a RTS frame from node $\mathrm{S}$, the location information about node $\mathrm{S}$ can be obtained by node $\mathrm{D}$. Then, node $\mathrm{D}$ calculates the relative position and selects the corresponding directional antenna to send the CTS frame. If this directional antenna is available in terms of DNAV table, node D begins to send the CTS frame with that directional antenna after the sub-channel is idle for a short inter-frame space (SIFS). Otherwise, the transmission of CTS frame is canceled. The information of node D's location is included in the CTS frame.

\section{3) CTS Receiving and DTA/ACK Transmission}

After sending the RTS frame omni-directionally, node $S$ waits for the CTS frame with omni-directional receiving mode. If it doesn't receive the CTS frame within a time limit, it will enter the judging state. If it receives the CTS frame successfully, it will select a proper directional antenna to send the following data packet based on the location of node D. During the reception of data packet, other directional antennas of node $\mathrm{D}$ are unavailable. After receiving the data packet, an ACK is transmitted using directional antenna. If node $S$ does not receive the ACK within a specified ACK_Timeout, it reschedules the packet transmission according to the given backoff rules.

Node S will enter the judging state if it does not receive the CTS frame within a given period. The specified operations in the judging state are shown in Fig. 1.

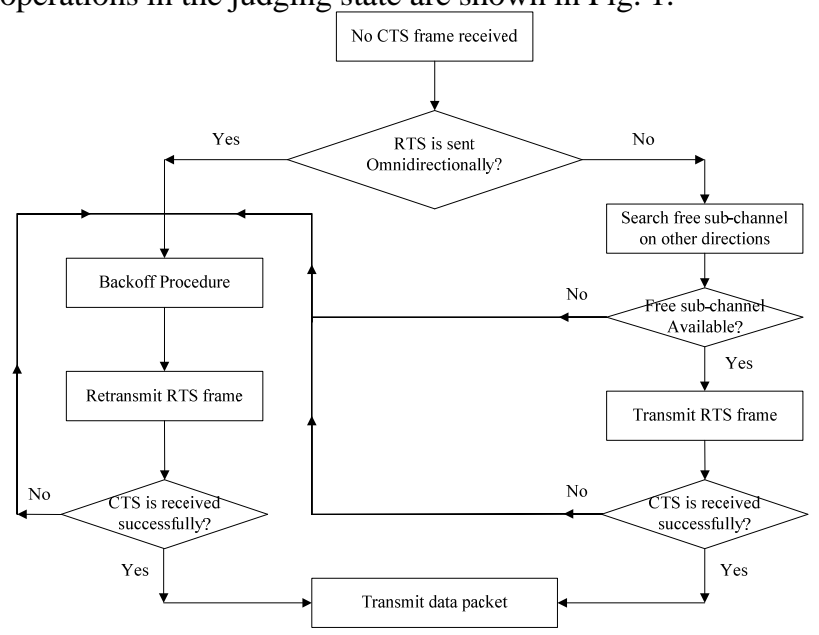

Figure 1. Flow chart of the operations in the judging state

Firstly, node S judges whether the RTS frame is transmitted with omni-directional mode. If the RTS is transmitted omni-directionally, the failure of the CTS reception is either caused by the collision of RTS frames or due to the busy state of node D. Therefore, node S starts the backoff procedure and doubles the contention window as the IEEE 802.11 MAC protocols. If the RTS is transmitted directionally, three possibilities leading to the loss of the CTS frame are as follows.

- Node D is out of the coverage of the directional antenna of node $\mathrm{S}$.

- Collisions of the RTS frames occur.

- $\quad$ Node D is busy transmitting with other node.

In order to determine the cause of the failure of the CTS reception under directional mode, we propose a method described as follows. Denote $N$ as the total number of directions supported by directional antennas. It is assumed that only $i(i<N)$ directions is not covered by transmission antenna of the RTS. Hence, node S searches the free subchannels for all the $i$ directions among the remaining subchannels according to the DNAV table. If a sub-channel is free and all of the $i$ directions on this sub-channel is available, node S sends RTS frames on this sub-channel with $i$ different directional antennas. If no free sub-channel exists for all the $i$ directions, while several sub-channels for $k(k<i)$ directions are free, the sub-channel with the biggest value of $k$, i.e. having the most available directions, is selected to transmit the RTS frame using $k$ directional antennas. In that case, 
when node S receives the CTS frame, it sends data packets with directional antenna on the current sub-channel after the channel is idle for a period of SIFS. Otherwise, node S starts the backoff procedure.

\section{SimULATION RESULTS}

In this part, the simulation results of the proposed new scheme are given. Furthermore, evaluation of the system performance, such as throughput, average access delay and packet dropping rate, are conducted between our scheme and other typical medium accessing schemes.

For simplicity of comparison, the scheme of multichannel accessing with directional antennas is denoted as DMCA (Directional Multichannel Access). The scheme of multichannel accessing with omni-directional antenna is denoted as OMCA (Omni-directional Multichannel Access) and that of a single channel accessing with omni-directional antenna is denoted as OSCA (Omni-directional Single Channel Access). In this paper, only the RTS/CTS mode is studied. The channel condition is assumed to be ideal in our simulation. The parameters used are listed in Table I.

TABLE I. THE SimUlation PARAMETERS

\begin{tabular}{|c|c|}
\hline Parameter & Value \\
\hline Channel rate (Mbps) & 11 \\
\hline MAC Header (bits) & 224 \\
\hline PHY Header (bits) & 129 \\
\hline DIFS ( $\mu s)$ & 34 \\
\hline SIFS ( $\mu s)$ & 16 \\
\hline Slot time $(\mu s)$ & 10 \\
\hline ACK (bits) & 134 \\
\hline RTS (bits) & 182 \\
\hline CTS (bits) & 134 \\
\hline Retry limit & 7 \\
\hline
\end{tabular}

The saturation throughput of the three schemes for different initial contention window size $W$ is shown in Fig. 2. The number of the sub-channel is 8 and the data packet length is 4092 bit. It can be seen that DMCA outperforms other schemes significantly. In the case of a contention window size of 24, DMCA achieves about 50\% higher throughput than OSCA and 16\% higher than OMCA. The reason is that the utilization of space and frequency spectrum is improved due to directional antennas and multichannel access, which can support more simultaneous transmission pairs. With the increasing of the minimum contention window, the throughput of both DMCA and OMCA boosts firstly, and then decreases. When the contention window $W$ increases from 8 to 24, the probability of collision can be reduces by increasing the backoff time. But for larger contention window, the directional antennas and the multichannel access mechanism play a smaller role to reduce packet collisions. On the contrary, the oversized contention window will lead to a decline in the channel utilization.

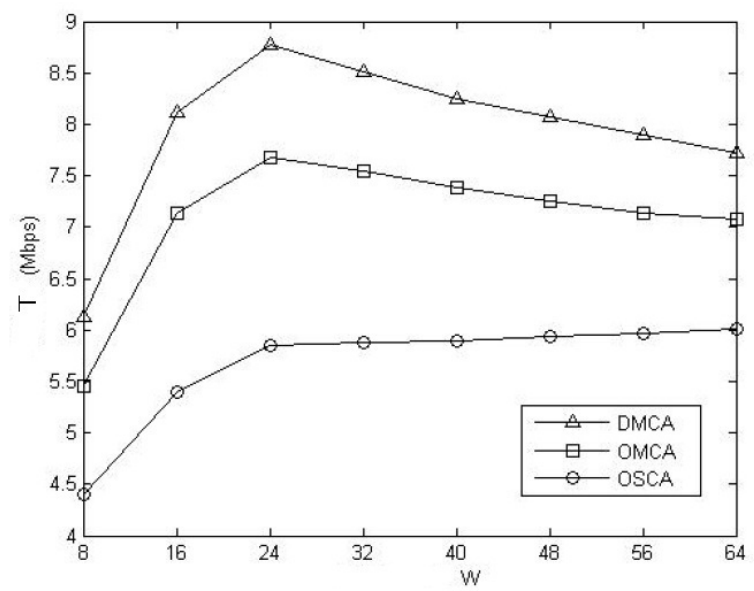

Figure 2. Throughput of the three access schemes in saturated system

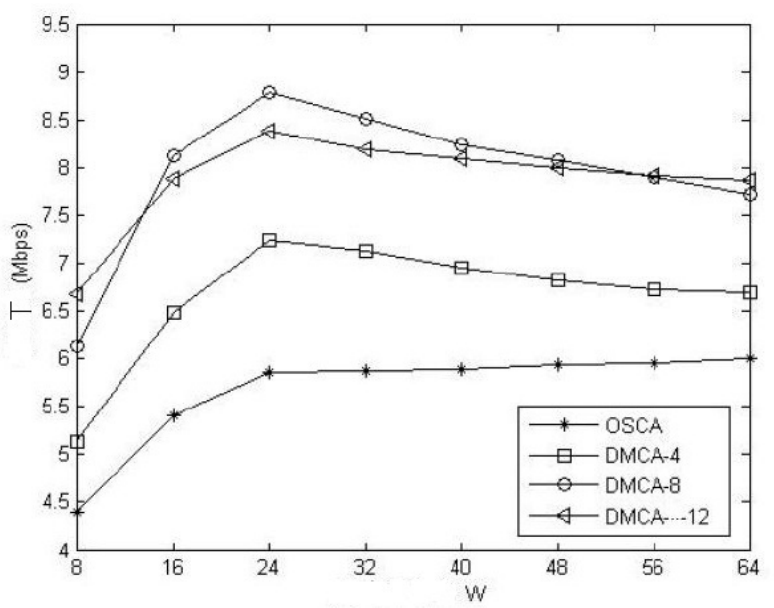

Figure 3. Throughput for different number of sub-channels

Fig. 3 presents the throughput of DMCA with different number of sub-channels in unsaturated conditions and then compares it with the OSCA scheme. The number of subchannels is set to 4, 8 and 12. Generally, the system throughput boosts as the increasing number of sub-channels. However, the performance of 12 sub-channels is a bit inferior to that of 8 sub-channels. The reasons are as follows. With the increasing number of sub-channels, the bandwidth of each sub-channel reduces. As a result, the transmission period of each data packet is extended and most of the nodes might be in transmitting or receiving state for a long time. In DMCA scheme, the backoff counter decreases only when the channel is free, so the backoff procedure is prolonged. Therefore, too many sub-channels may lead to long frozen time for the backoff counters and deteriorates the throughput performance.

The average access delays of three access schemes for different minimum contention window size $W$ are given in Fig. 4. The access delay is defined as the time period from the node's contending channel to the successful reception of the data packet. Then, the average delay can be obtained by averaging the sum of the delay of all the successfully transmitted packets. As expected, DMCA has the lowest 
access delay. For larger minimum contention window, packet collisions can be avoided, which saves the time spent on retransmissions.

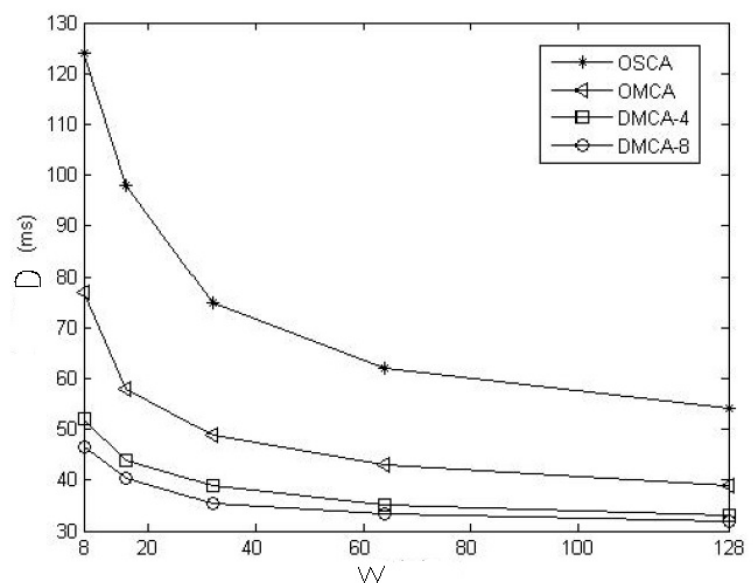

Figure 4. Access delay (D) of different access schemes in saturated system

In Fig. 5, we compare the packet dropping rate of different access schemes. If the number of retransmission time exceeds the retry limit, the date packet will be dropped. It can be found that DMCA improves the performance of packet dropping rate significantly. For example, DMCA achieves a $25 \%$ lower packet dropping rate at best than OMCA.

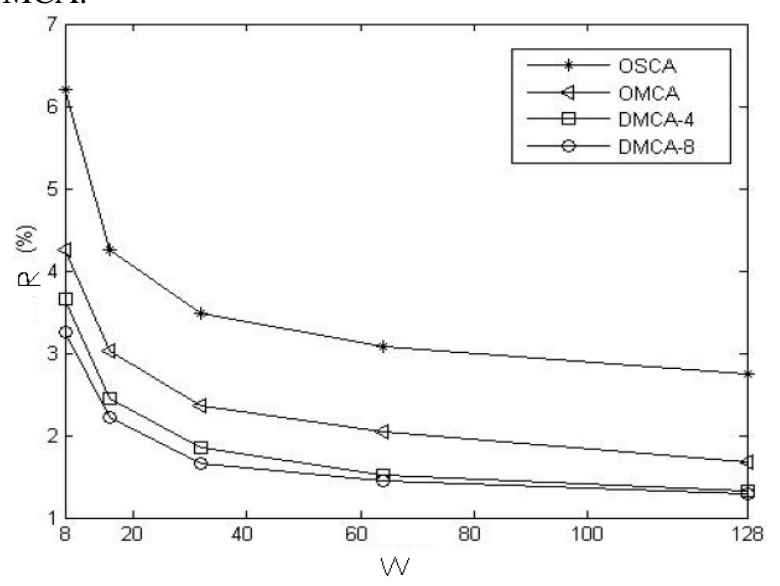

Figure 5. Packet dropping rate (R) of different access schemes

\section{CONCLUSION}

Using the omni-directional antenna transceivers and the single-channel access cannot take full advantage of the frequency spectrum and the space resources in the traditional MAC protocol. To solve the problem, we propose a solution of the multi-channel access with directional antennas under the condition of the unknown node location information. The new scheme improves the resource reuse rate in the spatial and frequency spectrum, increases the system throughput significantly, reduces the end-to-end delay caused by the channel contention, and reduces the packet dropping rate caused by retransmissions. As the advantage is without the prior informed node location information, our solution reduces the device costs and improves the feasibility.

\section{REFERENCES}

[1] D. Lal, R. Toshniwal, R. Radhakrishna, D. Agrawal and J. Caffery, “A Novel MAC Layer Protocol for Space Division Multiple Access in Wireless Ad Hoc Networks," in Proc.lC3N, 2002.

[2] M. Sanchez, T. Giles and J. Zander, "CSMA/CA with Beamforming Antennas in Multi-Hop Packet Radio," in Proc. Swedish Workshop on Wireless Ad Hoc Networks, pp.63-69. Mar., 2001.

[3] H. Singh and S. Singh, "DOA-ALOHA: Slotted ALOHA for Ad Hoc Networking Using Smart Antennas,” in Proc. Vehicular Technology Conf. (VTC), Fall 2003.

[4] Y. Ko, V. H. Vaidya, "Medium Access Control Protocols Using Directional Antennas in Ad Hoc Networks," in Proc. IEEE Inforcom, vol. 1, Mar. 2000.

[5] K. Kobayashi and M. Nakagawa, "Spatially Divided Channel Scheme Using Sectored Antennas for CSMA/CA-Directional CSMA/CA,” in Proc. Int'l Symp. Personal Indoor and Mobile Radio Comm. Conf., 2000.

[6] J. So and N. H. Vaidya, "Multi-Channel MAC for Ad Hoc Networks: Handling Multi-channel Hidden Terminals Using a Single Transceiver," in Proc ACM International Symposium on Mobile Ad Hoc Networking and Computing, May 2004, pp. 222-233.

[7] X. Yang, N. H. Vaidya, and P. Ravichandran, "Split-Channel Pipelined Packet Scheduling for Wireless Networks,” IEEE Trans. Mobile Comput., vol. 5, no. 3, pp. 240-257, Mar. 2006.

[8] K. Bai and J. Zhang, "Opportunistic Multichannel Aloha: Distributed Multiaccess Control Scheme for OFDMA Wireless Networks,” IEEE Trans.Vehicular Tech., col. 55, no. 3, May, 2006.

[9] J. Deng, Y. S. Han and Z. J. Haas, “Analyzing Split Channel Medium Access Control Schemes,” IEEE Trans. Wireless Commun., vol. 5, no. 5, pp. 967-971, May, 2006.

[10] IEEE Standard for Wireless LAN Medium Access Control (MAC) and Physical Layer (PHY) Specifications, Nov. 1997. 